\title{
The Golden Ratio and Viète's Formula
}

Esther M. García-Caballero, Samuel G. Moreno and Michael P. PRophet

Abstract. Viète's formula uses an infinite product to express $\pi$. In this paper we find a strikingly similar representation for the Golden Ratio.

Key words and phrases: Golden Ratio, nested radicals, Viète-like formulas.

ZDM Subject Classification: A30, G30, I30.

\section{Introduction}

As teachers, we constantly remind our students of the importance of mathematics, primarily due to its ubiquity in nature and science. And perhaps nothing better exemplifies this prevalence than both the Golden Ratio $\phi$ and the Archimedes' constant (also, Ludolph's constant) $\pi$. In this paper, we show an interesting connection between these values by expressing $\phi$ from a "slight" variation on a classical representation of $\pi$.

The formula we will encounter involves an infinite product of nested square roots. As part of our presentation, we will help instructors demystify these expressions for students through two Learning Activities. The Learning Activities provide instructors with a set of suggestions that give a "hands-on" approach, first to an infinite nesting of square roots that reproduces the Golden Ratio $\Phi$,

This work was partially supported by Junta de Andalucía, Research Group FQM 0178.

Copyright (C) 2014 by University of Debrecen 
and then to an expression for $\pi$ that utilizes an infinite product (the so-called Viète's formula).

1.1. Learning Activity I: Infinite encounters with ratios and square roots

In this Activity we will develop expansions for the Golden Ratio that involve infinite "nestings" of common mathematical operations.

Begin with a short historical remark on the origin of the Golden Ratio: from Euclid's Elements [4, Book VI, Definition 3] we have the first known definition of the Golden Ratio which, in fact, is referred to as extreme and mean ratio: "A straight line is said to have been cut in extreme and mean ratio when, as the whole line is to the greater segment, so is the greater to the less."

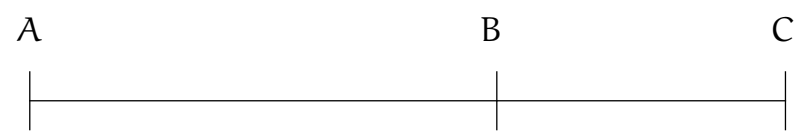

Figure $1 . B$ cuts $A C$ in the Golden Ratio if $A C / A B=A B / B C$.

With students working individually or in groups, ask them to draw on paper a line segment $A C$ and them estimate the location of a third point $B$ such that $A C / A B=A B / B C$ holds. Now, if they denote by $\phi$ the length of the "greater segment" $A B$, and they let 1 (unit) corresponds to the length of the "less one" BC, they should then establish the equality $(\phi+1) / \phi=\phi / 1$, and derive the equation

$$
\phi^{2}-\phi-1=0
$$

which has a first (positive) solution $\phi=(1+\sqrt{5}) / 2$ (here they have the standard expression for the Golden Ratio), and a second (negative) one $(1-\sqrt{5}) / 2=$ $-1 / \phi=1-\phi$.

Now ask students to discover more "exotic" expressions for $\phi$ (the purpose is to show them how easily some infinite expansions for this constant can be obtained). By first clearing the term $\phi^{2}$ in (1) and then either taking square roots or dividing by $\phi$, they should derive the two equations

$$
\phi=\sqrt{1+\phi}
$$

and

$$
\phi=1+\frac{1}{\phi}
$$


The next step is to show them that these equations can be used to create new equations via iteration. Show, for example, that one iteration of (2) gives

$$
\phi=\sqrt{1+\sqrt{1+\phi}}
$$

while an iteration of (3) gives

$$
\phi=1+\frac{1}{1+\frac{1}{\phi}} .
$$

Indeed, by continuing this iteration process (and ensuring convergence), show students that we are led to the two best-known infinite expansions for $\phi$ :

$$
\sqrt{1+\sqrt{1+\sqrt{1+\cdots}}}=\phi=1+\frac{1}{1+\frac{1}{1+\frac{1}{\ddots}}} .
$$

It is worth pointing out that the following "proofs without words" provide an easy way to see the convergence of both expansions in (4) (see Fig. 2 below)
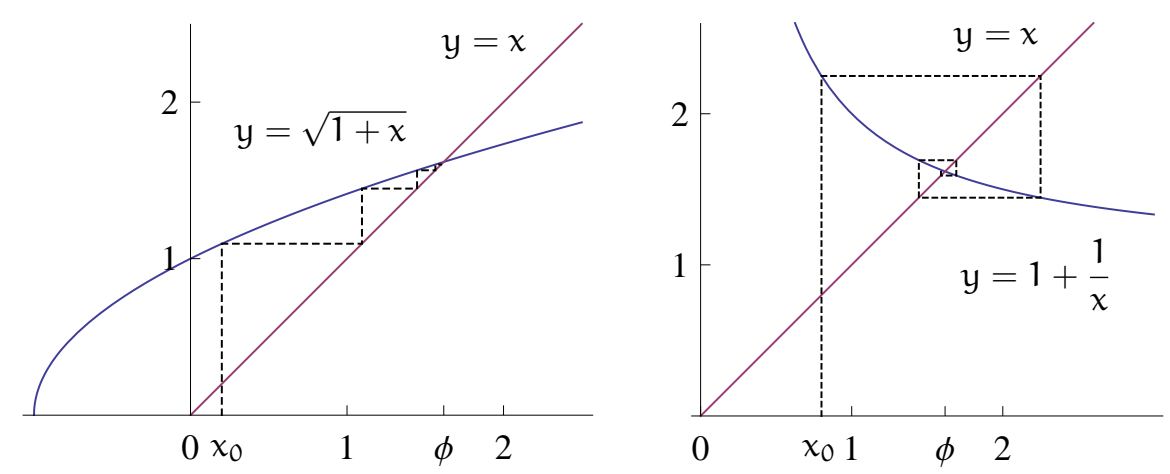

Figure 2. The recurrences $x_{n+1}=\sqrt{1+x_{n}}$ (left) and $x_{n+1}=1+1 / x_{n}$ (right).

Now, the instructor can suggest that students try "mixing" the iterations of (2) and (3) to obtain new expansions, one of the simplest being

$$
\phi=\sqrt{1+\phi}=\sqrt{1+1+\frac{1}{\phi}}=\sqrt{2+\frac{1}{\sqrt{1+\phi}}}=\sqrt{2+\frac{1}{\sqrt{1+1+\frac{1}{\phi}}}}
$$




$$
\begin{aligned}
& =\sqrt{2+\frac{1}{\sqrt{2+\frac{1}{\phi}}}} \\
& =\sqrt{2+\frac{1}{\sqrt{2+\frac{1}{\sqrt{2+\frac{1}{\ddots}}}}}}
\end{aligned}
$$

which is equivalent with the recurrence equation $x_{n+1}=\sqrt{2+1 / x_{n}}, n \geq 1$, with $x_{1}=\sqrt{2}$. As another example, consider working with students to discover the infinite pattern that results from the iterative scheme

$$
\text { ((2), (2), (3), (2), (2), (3), (2), (2), (3), ..), }
$$

which gives us

$$
\begin{aligned}
& \phi=\sqrt{1+\phi}=\sqrt{1+\sqrt{1+\phi}}=\sqrt{1+\sqrt{2+\frac{1}{\phi}}} \\
& =\sqrt{1+\sqrt{2+\frac{1}{\sqrt{1+\phi}}}}=\sqrt{1+\sqrt{2+\frac{1}{\sqrt{1+\sqrt{1+\phi}}}}} \\
& =\sqrt{1+\sqrt{2+\frac{1}{\sqrt{1+\sqrt{2+\frac{1}{\phi}}}}}}
\end{aligned}
$$

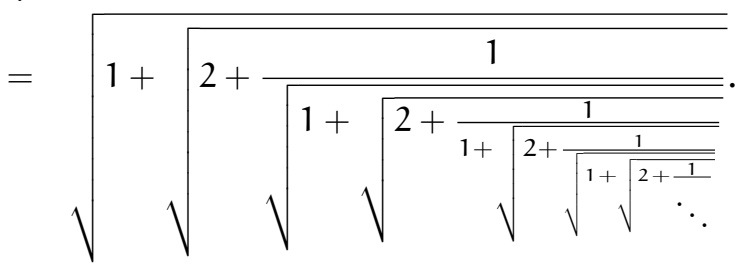

\subsection{Learning Activity II: The infinite product in Viète's Formula}

In this Activity we will informally verify geometric observations that allow us to express the area of a circle, and hence $\pi$, as a repeated product of (once again) 
nested square roots. Begin with a short historical remark on the origin of Viète's Formula: in 1593, the French amateur mathematician François Viète derived a formula for the exact value for $\pi$, by means of the first recorded infinite product in mathematics. Viète's technique relied on the following geometric observation:

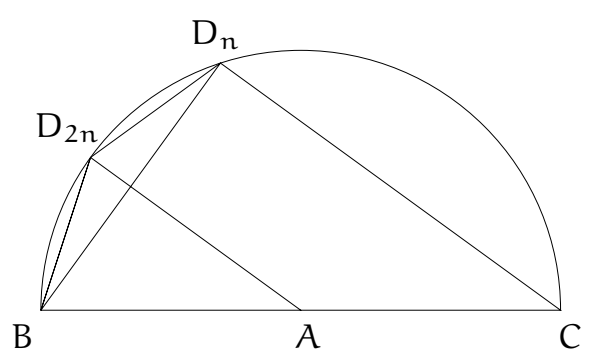

Figure 3. Viète's geometric construction.

Proposition 1. [6, Propositio I] and [1, p.754-755] If $A_{n}$ stands for the area of the regular polygon with $n$ sides (of length $B_{n}$ ) and $A_{2 n}$ stands for the area of the regular polygon with $2 n$ sides (of length $B D_{2 n}$ ), then $A_{n} / A_{2 n}=a_{n} / d$, being $\mathrm{a}_{\mathrm{n}}=\mathrm{D}_{\mathrm{n}} \mathrm{C}$ the so-called apotome of the side $\mathrm{BD}_{\mathrm{n}}$ and $\mathrm{d}$ the diameter of the circle.

Working individually or in groups, ask students to check this result for the $\mathrm{n}=4$ case. This activity works best when students can employ a ruler and compass. Alternatively, the instructor may verify this in front of the class using traditional drawing tools or computer software projected on a classroom screen.

With the following Proposition in mind, demonstrate how the above Proposition can be "leveraged" to create a repeating product for ratios of the form $A_{n} / A_{2^{k+1} n}$. Begin by showing the $k=1$ case:

$$
\frac{A_{n}}{A_{2^{2} n}}=\frac{A_{n}}{A_{2 n}} \cdot \frac{A_{2 n}}{A_{2^{2} n}}=\frac{a_{n} a_{2 n}}{d^{2}}
$$

Then, by using induction, students can see why Viète's next step of construction is valid:

Proposition 2. [6, Propositio II] and [1, p.755-756] For each nonnegative integer $\mathrm{k}$,

$$
\frac{A_{n}}{A_{2^{k+1} n}}=\frac{a_{n} a_{2 n} a_{2^{2} n} \cdots a_{2^{k} n}}{d^{k+1}},
$$


where for each $j=0,1, \ldots, k, a_{2^{j} n}=D_{2^{j} n} C$ is the apotome of the side $B_{2^{j} n}$.

Remind students that the above result holds for all k, so we may consider the limit as $k$ tends to infinity. Check students' insight asking them what the meaning of $A_{\infty}$ should be. And so, with $n=4$ (the square inscribed in the circle), students should see why Viète obtained:

Corollary 3. [6, Corollarivm] and [1, p.756-757] If $\mathrm{A}_{\infty}$ denotes the area of the circle, which can be considered as the regular polygon with infinitely many sides, then

$$
\frac{A_{4}}{A_{\infty}}=\frac{a_{4}}{d} \frac{a_{8}}{d} \frac{a_{16}}{d} \cdots=\prod_{i=0}^{\infty} \frac{a_{2^{i+2}}}{d} .
$$

In Corollary 3 we have used modern terminology, avoiding the problematic "highest power of the diameter", $\mathrm{d}^{\infty}$, used by Viète. Let us say also that Viète's original Corollarivm consists in a paraphrased version of formula

$$
\frac{A_{4}}{A_{\infty}}=\frac{l_{4}}{\prod_{i=1}^{\infty} a_{2^{i}+2}},
$$

where $l_{4}=a_{4}$ is the side of the square. Let us also comment that after Corollary 3 Viète wrote, without additional explanation, that if $\mathrm{d}=2$, then $\mathrm{a}_{4}=\sqrt{2}$, $a_{8}=\sqrt{2+\sqrt{2}}, a_{16}=\sqrt{2+\sqrt{2+\sqrt{2}}}$ and "so on in that progression". This comment should lead, after using formula (5), to the so-called Viète's infinite product

$$
\frac{2}{\pi}=\frac{\sqrt{2}}{2} \frac{\sqrt{2+\sqrt{2}}}{2} \frac{\sqrt{2+\sqrt{2+\sqrt{2}}}}{2} \cdots .
$$

Therefore, as the final step in the understanding of Viète's construction, students will need expressions for $a_{4}, a_{8}, a_{16}, \ldots$ They are left with the task of verifying that for $n \geq 4$,

$$
a_{2 n}=\sqrt{\frac{d^{2}}{2}+\frac{d}{2} a_{n}} .
$$

This will give, for $d=2$ and for the (obvious) value $a_{4}=\sqrt{2}$, the sequence $a_{8}=\sqrt{2+\sqrt{2}}, a_{16}=\sqrt{2+\sqrt{2+\sqrt{2}}}$, etc. And from (5) one gets (6). 


\section{Main result}

This section is devoted to establish a Viéte-like infinite product for representing the Golden Ratio $\phi$. To the best of our knowledge, the formula we give is new and it connects rather strikingly two such different constants as $\phi$ and $\pi$.

For sake of comparison, we begin by noting that from Viéte's infinite product (6) we get the following formula for $\pi$ :

$$
\pi=2 \prod_{k=1}^{\infty}(2 / \underbrace{\sqrt{2+\sqrt{2+\cdots+\sqrt{2}}}}_{\mathrm{k} \text { square roots }}) .
$$

The denominators of each one of the factors in the above infinite product consist of nested square roots of 2 with plus signs inside; it is interesting to consider the result that (reasonable) changes make to this product. For example, consider the product of alternating nested square roots of 2 given by

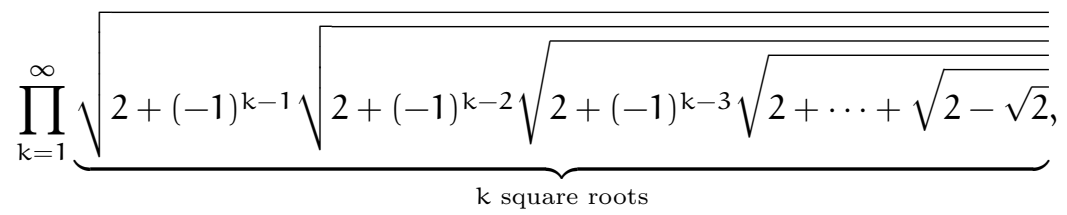

which expands as

$$
(\sqrt{2})(\sqrt{2-\sqrt{2}})(\sqrt{2+\sqrt{2-\sqrt{2}}})(\sqrt{2-\sqrt{2+\sqrt{2-\sqrt{2}}}})
$$

In the following result, we show that not only does this product converge (when grouping in a suitable manner), but it can be used to build a Viete-like formula for $\phi$, similar in form to (7).

\section{Theorem 4.}

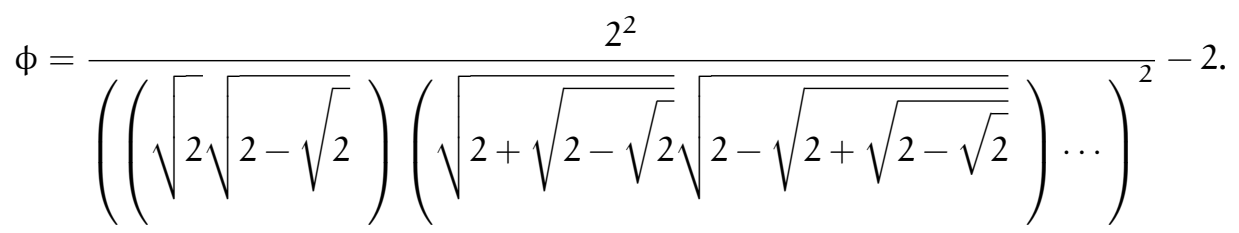


Proof. We begin by defining $\rho_{1}=\sqrt{2}$ and $\rho_{k+1}=\sqrt{2+(-1)^{k} \rho_{k}}$ for $k \geq 1$ and then, with $\sigma_{2 k}=\sqrt{2+\rho_{2 k-1}}$, claim that, for $n=1,2, \ldots$,

$$
2=\left(\prod_{k=1}^{n}\left(\rho_{2 k-1} \rho_{2 k}\right)\right) \sigma_{2 n}
$$

But this is easily proven using induction:

(i) First, the base case: we need to verify that (8) holds for $n=1$. Effectively,

$$
\rho_{1} \rho_{2} \sigma_{2}=\sqrt{2}(\sqrt{2-\sqrt{2}} \sqrt{2+\sqrt{2}})=(\sqrt{2})^{2}=2
$$

which is our first claim.

(ii) Second, the induction step: let $m$ be a positive integer and assume that (8) holds for $n=m$, so $\left(\prod_{k=1}^{m}\left(\rho_{2 k-1} \rho_{2 k}\right)\right) \sigma_{2 m}=2$. We need to show that (8) holds for $n=m+1$. To this purpose, we note that

$$
\left(\prod_{k=1}^{m+1}\left(\rho_{2 k-1} \rho_{2 k}\right)\right) \sigma_{2 m+2}=\left(\prod_{k=1}^{m}\left(\rho_{2 k-1} \rho_{2 k}\right)\right)\left(\rho_{2 m+1} \rho_{2 m+2} \sigma_{2 m+2}\right) .
$$

By using the definitions of $\rho_{k}$ and $\sigma_{2 k}$, we get

$$
\begin{aligned}
\rho_{2 m+1} \rho_{2 m+2} \sigma_{2 m+2} & =\rho_{2 m+1} \sqrt{2-\rho_{2 m+1}} \sqrt{2+\rho_{2 m+1}}=\rho_{2 m+1} \sqrt{4-\rho_{2 m+1}^{2}} \\
& =\sqrt{2+\rho_{2 m}} \sqrt{4-\left(2+\rho_{2 m}\right)}=\sqrt{2+\rho_{2 m}} \sqrt{2-\rho_{2 m}} \\
& =\sqrt{4-\rho_{2 m}^{2}}=\sqrt{4-\left(2-\rho_{2 m-1}\right)}=\sqrt{2+\rho_{2 m-1}} \\
& =\sigma_{2 m} .
\end{aligned}
$$

If we first substitute (10) into (9), and next we use the induction hypothesis, we have

$$
\left(\prod_{k=1}^{m+1}\left(\rho_{2 k-1} \rho_{2 k}\right)\right) \sigma_{2 m+2}=\left(\prod_{k=1}^{m}\left(\rho_{2 k-1} \rho_{2 k}\right)\right) \sigma_{2 m}=2
$$

so we are done.

Now our goal is to express $\sigma_{2 n}$ (above) in terms of the trigonometric values $\cos \pi x$, for properly chosen $x$. Specifically, using the main Theorem (and notation) from [5], we have for $k=2,3, \ldots$,

$$
\cos \left(f\left(b_{k}, \ldots, b_{1}\right)\right)=R\left(b_{k}, \ldots, b_{1}\right)
$$


where $b_{1} \in[-2,2]$ and $b_{i} \in\{-1,0,1\}$ for $i>1$,

$$
\begin{gathered}
f\left(b_{k}, \ldots, b_{1}\right)= \\
=\left(\frac{1}{2}-\frac{b_{k}}{4}-\frac{b_{k} b_{k-1}}{8}-\frac{b_{k} b_{k-1} b_{k-2}}{16}-\cdots-\frac{b_{k} b_{k-1} b_{k-2} \cdots b_{1}}{2^{k+1}}\right) \pi
\end{gathered}
$$

and

$$
R\left(b_{k}, \ldots, b_{1}\right)=\frac{b_{k}}{2} \sqrt{2+b_{k-1} \sqrt{2+b_{k-2} \sqrt{2+\cdots+b_{2} \sqrt{2+2 \sin \left(\frac{b_{1} \pi}{4}\right)}}}}
$$

Note that

$$
\sigma_{2 n}=\underbrace{\sqrt{2+\sqrt{2+\sqrt{2-\sqrt{2+\sqrt{2-\sqrt{2+\cdots-\sqrt{2}}}}}}}}_{2 n \text { square roots }}
$$

and thus using (13) we may write $\sigma_{2 n}=2 \mathrm{R}(1,1,1,-1,1,-1, \ldots, 1,-1)$. Connecting this expression back to (11) and (12), we obtain

$$
\begin{aligned}
\sigma_{2 n} & =2 \cos (f(1,1,1,-1,1, \ldots,-1)) \\
& =2 \cos \left(\left(\frac{1}{2}-\frac{1}{2^{2}}-\sum_{j=3}^{2 n+1} \frac{(-1)^{\left\lfloor\frac{j+1}{2}\right\rfloor}}{2^{j}}\right) \pi\right) \\
& =2 \cos \left(\frac{4^{n}-(-1)^{n}}{4^{n}} \frac{\pi}{10}\right),
\end{aligned}
$$

where $\lfloor\cdot\rfloor$ denotes the integer-part function. And since the cosine function is continuous, we find

$$
\begin{aligned}
& \lim _{n \rightarrow \infty} \sigma_{2 n}=\lim _{n \rightarrow \infty}\left(2 \cos \left(\frac{4^{n}-(-1)^{n}}{4^{n}} \frac{\pi}{10}\right)\right)= \\
= & 2 \cos \left(\lim _{n \rightarrow \infty}\left(\frac{4^{n}-(-1)^{n}}{4^{n}} \frac{\pi}{10}\right)\right)=2 \cos (\pi / 10) .
\end{aligned}
$$

As a final step, we see from $[2]$ that $\sin (\pi / 10)=(\sqrt{5}-1) / 4=1 /(2 \phi)$. This implies $2 \cos (\pi / 10)=\sqrt{2+\phi} \neq 0$. 
Now we have to combine the previous results to get

$$
2 \frac{1}{\sqrt{2+\phi}}=2 \frac{1}{\lim _{n \rightarrow \infty} \sigma_{2 n}}=2 \lim _{n \rightarrow \infty}\left(\frac{1}{\sigma_{2 n}}\right) .
$$

But taking limits in (8) we can rewrite the left hand side in (15) as

$$
\begin{aligned}
\frac{2}{\sqrt{2+\phi}} & =\lim _{n \rightarrow \infty}\left(\left(\prod_{k=1}^{n}\left(\rho_{2 k-1} \rho_{2 k}\right)\right) \sigma_{2 n}\right) \lim _{n \rightarrow \infty}\left(\frac{1}{\sigma_{2 n}}\right) \\
& =\lim _{n \rightarrow \infty}\left(\left(\prod_{k=1}^{n}\left(\rho_{2 k-1} \rho_{2 k}\right)\right) \sigma_{2 n} \frac{1}{\sigma_{2 n}}\right) \\
& =\lim _{n \rightarrow \infty}\left(\prod_{k=1}^{n}\left(\rho_{2 k-1} \rho_{2 k}\right)\right)
\end{aligned}
$$

which, after clearing $\phi$, completes the proof.

REMARK 5. A remarkable feature of the formula given in Theorem 4 is that it expresses the Golden Ratio using only the integer 2 (whereas the well-known expansions in (4) use only the integer 1). We wonder if 2 is the largest integer for which this is possible. That is, for which positive integers $n>2$ can $\phi$ be expressed using $\mathfrak{n}$ exclusively?

REMARK 6. It is worth pointing out that although the sequence

$$
\left\{\prod_{k=1}^{n} \rho_{k}\right\}_{n=1}^{\infty}
$$

does not converge, its behavior is "rather acceptable" due to the fact (proved above) that

$$
\lim _{n \rightarrow \infty} \prod_{k=1}^{2 n} \rho_{k}=\lim _{n \rightarrow \infty} \prod_{k=1}^{n}\left(\rho_{2 k-1} \rho_{2 k}\right)=\frac{2}{\sqrt{2+\phi}}
$$

and also, as noted by a referee, by

$$
\lim _{n \rightarrow \infty} \prod_{k=1}^{2 n+1} \rho_{k}=\sqrt{\frac{4 \phi}{2 \phi-1}} .
$$


We left the interested readers the task of verifying this last expression. The same referee derived the alternative formula

$$
\frac{1}{\phi}=2-\frac{2^{2}}{\lim _{n \rightarrow \infty}\left(\prod_{k=1}^{2 n+1} \rho_{k}\right)^{2}}
$$

which bears some resemblance to our formula $\phi=\frac{2^{2}}{\lim _{n \rightarrow \infty}\left(\prod_{k=1}^{2 n} \rho_{k}\right)^{2}}-2$.

REMARK 7. Note that from the fact that $\lim _{n \rightarrow \infty} \sigma_{2 n}=\sqrt{2+\phi}$, and taking into account the identity (14), we get

$$
\phi=\frac{1+\sqrt{5}}{2}=\sqrt{2+\sqrt{2-\sqrt{2+\sqrt{2-\sqrt{2+\cdots}}}}}
$$

which is an amazing relation appeared in [3]. Of course, by the way in which the above nested radical is obtained, it must be understood as the limit of the sequence $\left\{\sigma_{2 n}^{2}-2\right\}_{n=1}^{\infty}=\left\{\rho_{2 n-1}\right\}_{n=1}^{\infty}$, that is, the limit of

$\rho_{1}=\sqrt{2}, \quad \rho_{3}=\sqrt{2+\sqrt{2-\sqrt{2}}}, \quad \rho_{5}=\sqrt{2+\sqrt{2-\sqrt{2+\sqrt{2-\sqrt{2}}}}}, \ldots$

Finally, let us mention that the nested radical in (16) is one element of the set $S_{2}$ considered in [7].

\section{Acknowledgements}

We thank the referees for their useful suggestions and comments, which have improved the presentation of the paper.

\section{References}

[1] L. Berggren, J. Borwein and P. Borwein, Pi: A Source Book, 3rd ed., Springer-Verlag, New York, 2004. 
[2] M. Bicknell and V. E. Jr. Hoggatt, Golden triangles, rectangles, and cuboids, Fibonacci Quart. 7 (1969), 73-91.

[3] M. Cipolla, Intorno ad un radicale continuo, Period. Mat. L'inseg. Second. 5 (1908), 179-185.

[4] Euclid, Elements, 300 BC, http://alepho.clarku.edu/ djoyce/java/elements/elements.html.

[5] L. D. Servi, Nested Square Roots of 2, Amer. Math. Monthly 110 (2003), 326-330.

[6] F. Viète, Variorum de Rebus Mathematicis Responsorum Liber VIII, Capvt XVIII (1593), 398-400. See also [1] for both the original paper in Latin in p. 53-55, and for a translated version in p. 754-757.

[7] S. Zimmerman and C. Ho, On Infinitely Nested Radicals, Math. Mag. 81 (2008), $3-15$.

ESTHER M. GARCÍA-CABALLERO and SAMUEL G. MORENO

DEPARTAMENTO DE MATEMÁTICAS

UNIVERSIDAD DE JAÉN

23071 JAÉN, SPAIN

E-mail: emgarcia@ujaen.es

E-mail: samuel@ujaen.es

MICHAEL P. PROPHET

DEPARTMENT OF MATHEMATICS

UNIVERSITY OF NORTHERN IOWA

CEDAR FALLS, IA, USA

E-mail: prophet@math.uni.edu

(Received May, 2013) 